\section{Ileal cancer and erosions in the small intestine revealed by capsule endoscopy}

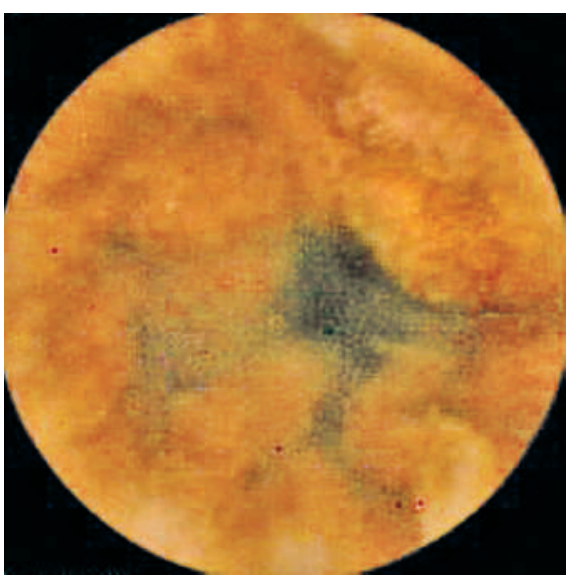

Figure 1 Erosions in the small intestine detected by the first capsule endoscopy.

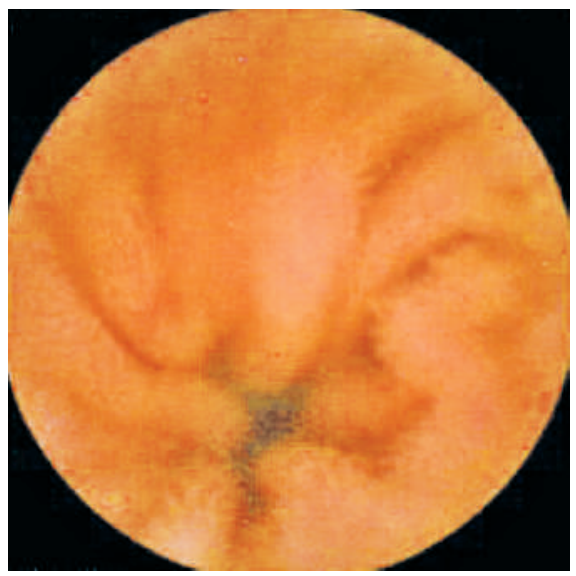

Figure 2 The second capsule endoscopy showed that there had been improvement in the small-intestinal lesions.

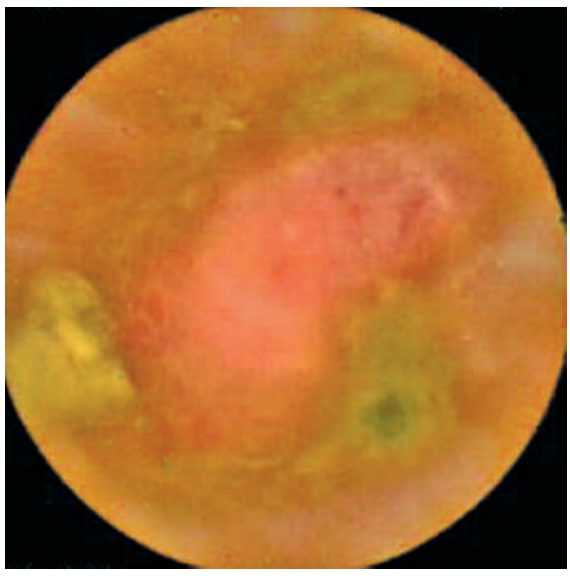

Figure 3 A protruding lesion near the terminal ileum that was detected by the second capsule endoscopy examination.

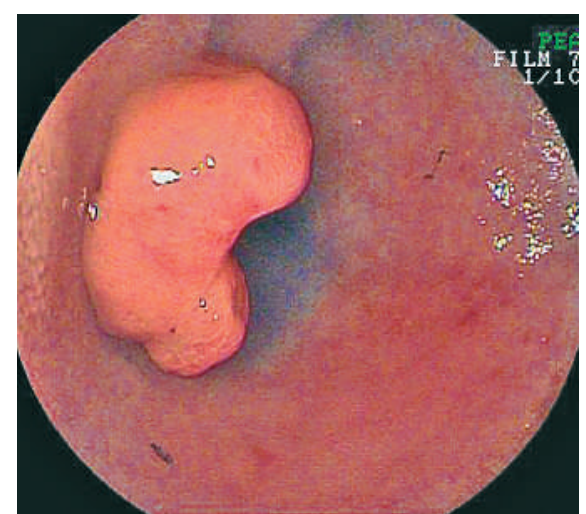

Figure 4 An ileal polyp found at double-balloon endoscopy.
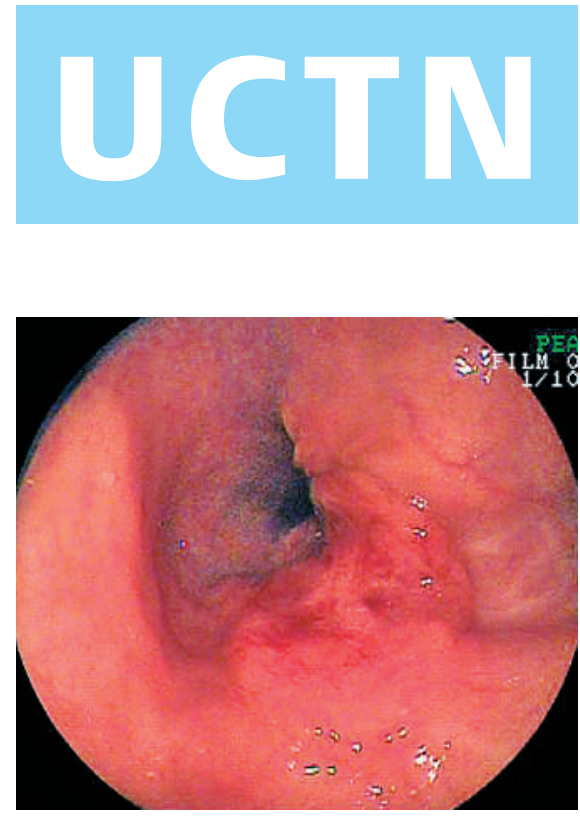

Figure 5 A malignant tumor in the ascending colon found at double-balloon endoscopy.

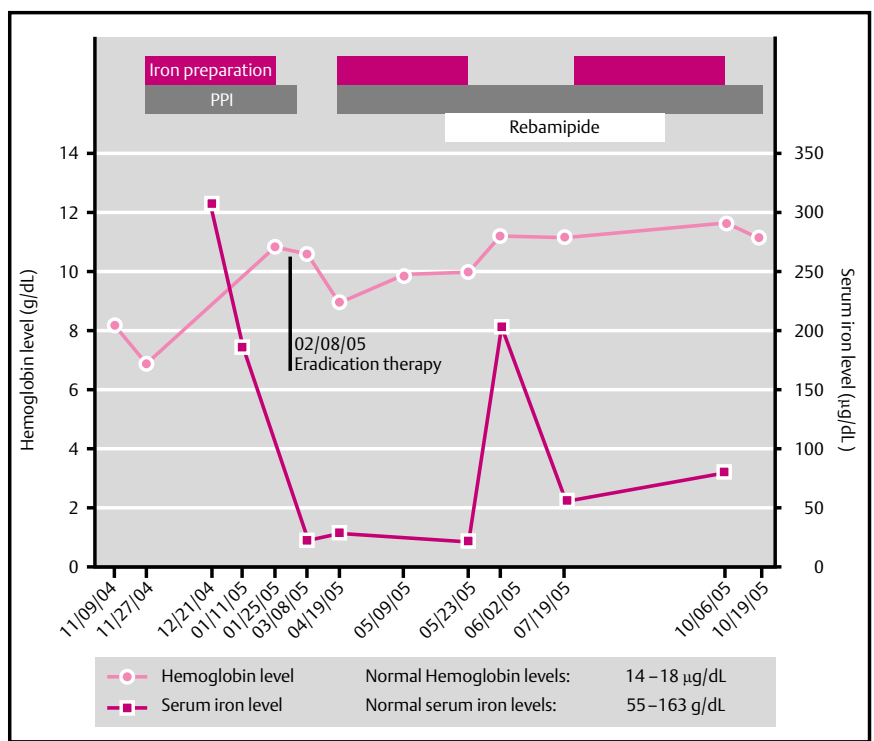

Figure 6 The patient's clinical course, showing the blood hemoglobin and serum iron levels, and the treatments he received over a period of nearly 1 year (PPI, proton-pump inhibitor).

An 80-year-old man with persistent anemia was suspected to have a hemorrhagic lesion in the small intestine and he underwent investigation by capsule endoscopy. This examination revealed several erosions and reddened lesions in the pelvic small intestine (Figure 1). On 17 May 2005 he was started on a prostaglandin inducer, rebamipide, at a daily dose of $300 \mathrm{mg}$, and although the iron preparation he was on was stopped, there was no decrease in his hemoglobin level. On 1 July a second capsule endoscopy examination revealed marked improvement the erosions and reddened lesions in the small intestine had resolved (Figure 2 ).
The second capsule endoscopy allowed observation nearly as far as the terminal ileum, where a protruding lesion was detected (Figure 3). Double-balloon endoscopy revealed a red type Ip polyp, about $30 \mathrm{~cm}$ proximal to the ileocecal valve (Figure 4). In addition, there was an advanced cancer resembling a type IIc early cancer in the ascending colon (Figure 5).

On 29 August 2005 the patient underwent surgery. The lesion in the large bowel was diagnosed as a moderately differentiated adenocarcinoma (ss, ly1, v1, n0)

DOI: $10.1055 / s-2006-944792$ 
and the lesion in the ileum as a well-differentiated adenocarcinoma with a component of tubular adenoma (sm1, ly0, v0). There was also a type IIc early cancer in the stomach and this lesion was resected endoscopically. The entire clinical course is summarized in Figure 6. It was not possible to determine whether this patient's anemia had been caused by the small-intestinal lesions or by the advanced large-bowel cancer. Rebamipide, a mucoprotective agent, might be effective for the management of patients with erosions or reddened lesions in the small intestine [1-3].

Capsule endoscopy proved useful for the diagnosis of the small-intestinal lesions in this patient, but we should be aware that this method cannot provide a diagnosis of lesions that the capsule cannot reach and that it can miss small lesions in stomach.

Endoscopy_UCTN_Code_CCL_1AC_2AC

\section{Y. Niwa', M. Nakamura', N. Omiya ${ }^{1}$,}

\section{A. Itoh', Y. Hirooka ${ }^{2}$, H. Goto ${ }^{1}$}

${ }^{1}$ Department of Gastroenterology, Nagoya Graduate School of Medicine, Nagoya, Japan

2 Department of Endoscopy, Nagoya University Hospital, Nagoya, Japan.

\section{References}

${ }^{1}$ Haruma K, Ito M. Review article: clinical significance of mucosal-protective agents: acid, inflammation, carcinogenesis and rebamipide. Aliment Pharmacol Ther 2003; 18 (Suppl 1): 153-159

2 Mizoguchi H, Ogawa Y, Kanatsu K et al. Protective effect of rebamipide on indometha- cin-induced intestinal damage in rats. J Gastroenterol Hepatol 2001; 16: 1112 - 1119

${ }^{3}$ Kojima M, Iwakiri R, Wu B et al. Effects of antioxidative agents on apoptosis induced by ischaemia-reperfusion in rat intestinal mucosa. Aliment Pharmacol Ther 2003; 18 (Suppl 1): 139-145

\section{Corresponding author}

\section{Y. Niwa, M.D.}

Department of Gastroenterology Nagoya Graduate School of Medicine 65 Tsuruma-cho

Showa-ku

Nagoya

466-8550

Japan

Fax: $\quad+81-52-744-2180$

Email: yniwa@med.nagoya-u.ac.jp 